\title{
Ultrasonic attenuation measurements on crystals which display persistent nonphotochemical ir spectral hole burning
}

\author{
H. Lengfellner* and A. J. Sievers \\ Laboratory of Atomic and Solid State Physics and Materials Science Center, \\ Cornell University, Ithaca, New York 14853 \\ (Received 26 November 1984)
}

\begin{abstract}
Temperature-dependent ultrasound-absorption measurements have been used to identify an $\mathrm{ReO}_{4}{ }^{-}$induced loss peak in alkali halide crystals. The measured shape is consistent with an activation process with barrier energies of $60 \mathrm{meV}$ for $\mathrm{RbBr}, 50 \mathrm{meV}$ for $\mathrm{KI}$, and $30 \mathrm{meV}$ for $\mathrm{RbI}$. The temperature dependence of the hopping time is compatible with the disappearence of the persistent ir spectral features of $\mathrm{ReO}_{4}^{-}$as the temperature is increased to $10 \mathrm{~K}$.
\end{abstract}

The observation of persistent nonphotochemical spectral hole $^{1}$ and antihole ${ }^{2}$ production in the $\nu_{3}$ vibrational mode absorption of $\mathrm{ReO}_{4}^{-}$molecules in alkali halide crystals has led to the supposition that the tetrahedral molecular ion has two rotationally equivalent configurations at the substitutional lattice site which are separated by a large energy barrier as a result of steric effects. The validity of this model is open to question since, for such an arrangement, ir active librational sidebands which should occur at frequencies near the $\nu_{3}$ value have not been observed. ${ }^{1}$

In this Rapid Communication we describe the first direct experimental identification of a two-configuration energy barrier associated with the $\mathrm{ReO}_{4}^{-}$alkali halide defect system. By measuring the absorption of $10-\mathrm{MHz}$ ultrasound in $\mathrm{RbBr}, \mathrm{KI}$, and $\mathrm{RbI}$ crystals doped with $\mathrm{ReO}_{4}{ }^{-}$molecules at temperatures from 1.8 to $100 \mathrm{~K}$, we have identified in each a loss peak near $50 \mathrm{~K}$. This impurity-induced peak stems from an activation process with a barrier height of $60 \mathrm{meV}$ for $\mathrm{RbBr}, 50 \mathrm{meV}$ for $\mathrm{KI}$, and $30 \mathrm{meV}$ for $\mathrm{RbI}$. The decreasing barrier height with increasing lattice constant may account for the observation that spectral holes are burned and erased most easily in the RbI host.

Ultrasonic measurements have been made on the Czochralski grown single crystals identified in Table I. The dopant concentrations listed correspond to the $\mathrm{KReO}_{4}$ added to the melt. In order to correct for the dopant concentra- tion gradient down a boule, infrared spectroscopy in the $\mathrm{ReO}_{4}-\left(\nu_{2}+\nu_{4}\right)$ combination band region has been used to obtain the relative dopant concentration.

Longitudinal ultrasound at $10 \mathrm{MHz}$ is generated and detected with an $x$-cut quartz transducer (hereafter referred to as TD) driven at its fundamental. Commercial equipment is used to produce, amplify, and detect the TD signal. A novel feature of our measurement is the TD-sample arrangement. The TD is pressed by a spring with a force of about $5 \mathrm{~N}$ onto the flat polished alkali halide surface; no bonding agent is used between the two. Because contact is made at a number of points a single unique path for the ultrasound does not exist and the output signal from the TD is very different from the bonded case.

The "noisy" signal shown in Fig. 1 is the amplified and rectified TD signal. A small, $50 \mu \mathrm{sec}$, time constant has been used for smoothing. The "noisy" structure on the TD signal remains unchanged during repeated oscilloscope traces. It is caused by the interference of sound waves impinging on the TD with a variety of phases. To extract the decay constant from this signal, the signal is processed with a boxcar containing two independent channels. The recording window, called $A$, of one channel is set to open with a delay of $\Delta t=1 \mathrm{msec}$ after window $B$ of the other channel opens, as indicated at the bottom of Fig. 1. For an exponential decay of the amplitude of the ultrasonic waves,

TABLE I. Sample information.

\begin{tabular}{|c|c|c|c|c|}
\hline $\begin{array}{c}\text { Sample } \\
\text { No. }\end{array}$ & $\begin{array}{c}\text { Boule } \\
\text { No. }\end{array}$ & $\begin{array}{l}\text { Host } \\
\text { crystal }\end{array}$ & $\begin{array}{c}\mathrm{KReO}_{4} \\
\text { concentration } \\
\text { (mole \%) }\end{array}$ & $\begin{array}{c}\text { Relative } \\
\text { concentration } \\
(\%)\end{array}$ \\
\hline 1 & $7701116 \mathrm{~W}$ & $\mathrm{RbBr}$ & 0.4 & \\
\hline 2 & $908206 \mathrm{~W}$ & $\mathrm{RbBr}$ & Pure & \\
\hline 3 & $7605201 \mathrm{~W}$ & KI & 0.6 & 100 \\
\hline 4 & $7602192 \mathrm{~W}$ & KI & 0.3 & 20 \\
\hline 5 & $\ldots$ & KI & 0.06 & 5 \\
\hline 6 & $7010301 \mathrm{~W}$ & KI & Pure & \\
\hline 7 & $\cdots$ & KI & Pure & \\
\hline 8 & $8011146 \mathrm{~W}$ & $\mathrm{RbI}$ & 0.8 (boule bottom) & 100 \\
\hline 9 & $8011146 \mathrm{~W}$ & RbI & 0.8 (boule top) & 20 \\
\hline 10 & $7904066 \mathrm{~W}-\mathrm{A} 2$ & $\mathrm{RbI}$ & 0.001 & \\
\hline 11 & $8012164 W-11$ & $\mathrm{KI}+2 \% \mathrm{NaI}$ & 0.05 & \\
\hline
\end{tabular}




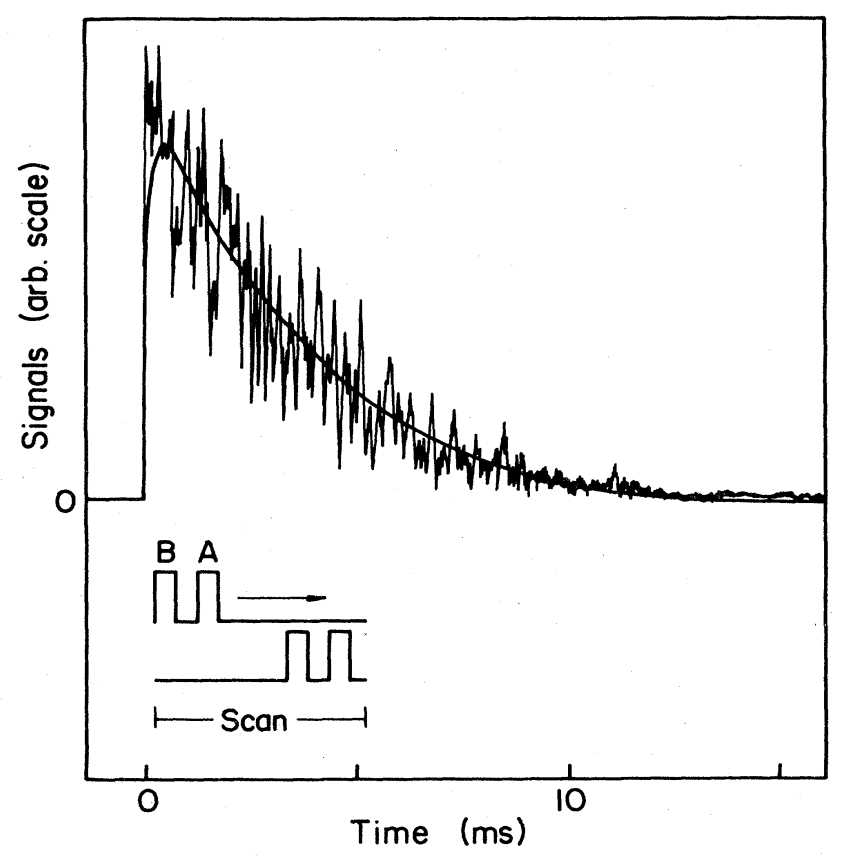

FIG. 1. Top: Decay of ultrasound in an alkali halide crystal at 2 K. A pulse is generated with a transducer at time zero. The oscilloscope traces show the time dependence of both the transducer signal ("noisy") and the optical signal (smooth). The data are for sample No. 11. Bottom: Data taking procedure for smoothing the "noisy" trace. See text for details.

one obtains for the absorption coefficient $\alpha$ (energy absorption)

$$
\alpha v=-\left(\frac{2}{\Delta t}\right) \ln \left(\frac{A}{B}\right),
$$

where $v$ is the sound velocity and $A / B$ the ratio of the two signals. To increase the precision of the measurement windows $A$ and $B$ are repeatedly scanned with fixed $\Delta t$ across the TD signal (indicated by scan in Fig. 1) using the boxcar "scan" mode. The boxcar output time constant is set to obtain an averaged $A / B$. The value $\alpha v$ is then obtained using Eq. (1).

A smooth signal trace is also shown in Fig. 1. This measurement of the ultrasonic amplitude decay with time does not make use of the TD as a detector; instead, it relies on the direct ir detection of the mechanical modulation by the ultrasound of the persistent spectral holes and antiholes in the $\nu_{3}$ vibrational mode. ${ }^{3}$ For this particular comparison the sample was cooled to $2 \mathrm{~K}$ since the persistent spectral effects only occur at low temperature.

The overall coincidence between the TD and the optical signal provides convincing evidence that our no-bond technique can be used to measure samples with small acoustic attenuation. From Fig. 1 an amplitude decay time of 4 msec (half height time) is obtained corresponding to an absorption coefficient $\alpha=10^{-3} \mathrm{~cm}^{-1}$. Because of diffraction limitations, it would be difficult to measure such small attenuation coefficients using the standard pulse-echo technique. ${ }^{4}$

A plot of $\alpha v$ versus the inverse temperature for a highly doped KI crystal (sample No. 3) is shown in Fig. 2. At a

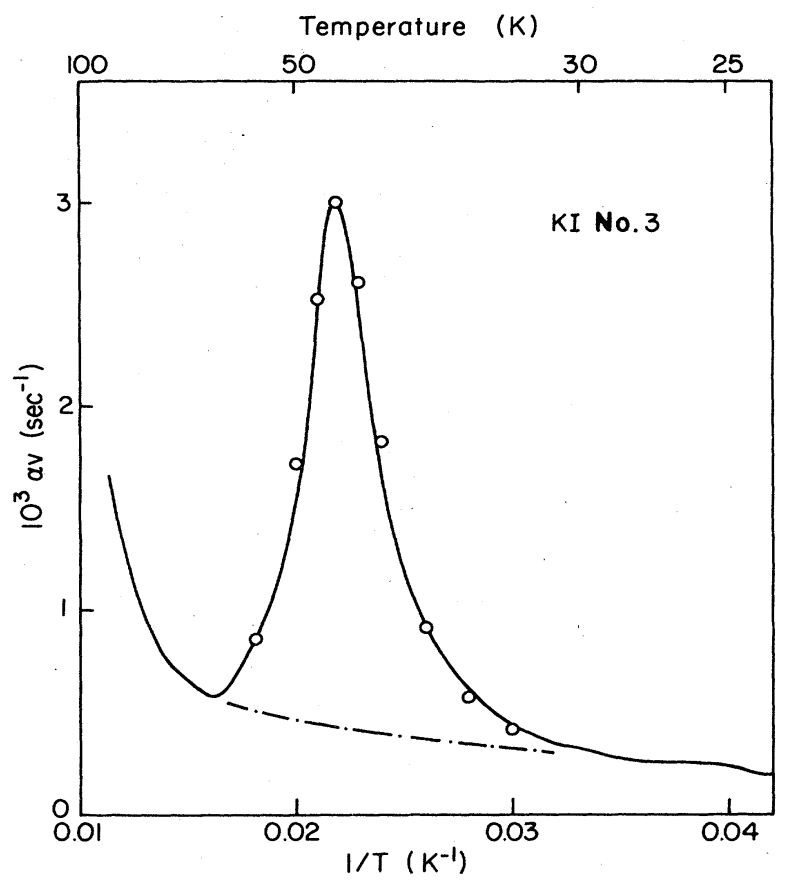

FIG. 2. Absorption peak for $10-\mathrm{MHz}$ ultrasound in $\mathrm{KI}: \mathrm{KReO}_{4}$ (sample No. 3). The product of absorption coefficient and sound velocity is plotted vs the inverse temperature. The circles are obtained by adding calculated values of $\alpha v$ (see text) to the estimated background (dash-dot line).

temperature $T(\alpha \max )=46 \mathrm{~K}$ a strong-absorption peak occurs. Additional measurements with samples No. 4, No. 5, No. 6, and No. 7 indicate that this absorption peak scales with the $\mathrm{KReO}_{4}$ concentration. Errors in the temperature measurement are estimated to be less than $\pm 2 \mathrm{~K}$ in the temperature region between 100 and $25 \mathrm{~K}$. The absolute value of $\alpha v$ is estimated to be accurate to $\pm 20 \%$.

The impurity-induced absorption peak in Fig. 2 can be fitted with a simple activation process. ${ }^{5}$ Let

$$
\alpha=\frac{A \omega \tau}{T\left(1+\omega^{2} \tau^{2}\right)}
$$

and

$$
\tau_{a}=\tau_{0 a} \exp (E / k T),
$$

where $A$ is the constant describing the absorption strength, $T$ is the sample temperature, and $\omega$ the ultrasound frequency. The absorption has a maximum value at the temperature $T(\alpha \max )$ where $\omega \tau=1$. By choosing a value $E$ for the activation energy the value $\tau_{0 a}$ for the relaxation time at high temperatures is fixed. The calculated values identified by circles in Fig. 2 have been obtained with a value $E=50$ $\mathrm{meV}\left(\tau_{0 a}=3 \times 10^{-14} \mathrm{sec}\right)$. These parameters are recorded in Table II.

Similar measurements for doped $\mathrm{RbBr}, \mathrm{KI}$, and $\mathrm{RbI}$ are shown in Fig. 3 (samples No. 1, No. 4, No. 8). The absorption peak shows two tendencies: with increasing lattice constant, the temperature $T(\alpha \max )$ shifts to lower temperature and the peak width increases. The parameters from fits of these data to Eqs. (2) and (3) also are given in Table II. Inspection of this table shows that the activation energy 
TABLE II. Experimental results for the activation process.

\begin{tabular}{|c|c|c|c|c|}
\hline $\begin{array}{l}\text { Host } \\
\text { crystal }\end{array}$ & $\begin{array}{l}\text { Lattice } \\
\text { constant } \\
\text { (A) }\end{array}$ & $\begin{array}{c}T(\alpha \max ) \\
(\mathrm{K})\end{array}$ & $\begin{array}{c}E \\
(\mathrm{meV})\end{array}$ & $\left(10^{-14} \mathrm{sec}\right)$ \\
\hline $\mathrm{RbBr}$ & 6.85 & 51 & 60 & 2 \\
\hline KI & 7.07 & 46 & 50 & 3 \\
\hline RbI & 7.34 & 42 & 30 & 800 \\
\hline
\end{tabular}

changes with lattice constant in a monotonic fashion.

Figure 4 shows lower temperature measurements from 1.8 to $20 \mathrm{~K}$ for three RbI samples (No. 8, No. 9, and No. 10) and, for comparison, the highly doped KI sample (No. 3). In contrast with $\mathrm{KI}$ and $\mathrm{RbBr}$ a strong absorption is observed in $\mathrm{RbI}$ at low temperatures. The temperature signature observed for the nominally pure RbI (No. 10) is somewhat different from the other two samples and is probably associated with an unwanted impurity. For samples No. 9 and No. 8 the value of $\alpha v$ at $2.5 \mathrm{~K}$ clearly increases with increasing $\mathrm{ReO}_{4}^{-}$concentration. At fixed concentration the absorption is relatively constant in magnitude up to $3 \mathrm{~K}$ but then decreases rapidly between 4 and $10 \mathrm{~K}$.

The appearance of this low-temperature absorption indicates that another degree of freedom of this defect-lattice system is being excited. The temperature signature is not consistent with a simple activation process nor with a phonon-assisted tunneling process. The activation process, represented by the dashed line $\left(E^{\prime}=0.5 \mathrm{meV}\right.$ and $\left.\tau_{0 a}^{\prime}=3 \times 10^{-5} \mathrm{sec}\right)$ in Fig. 4 does not agree with the data at the lowest temperatures. For a phonon-assisted tunneling

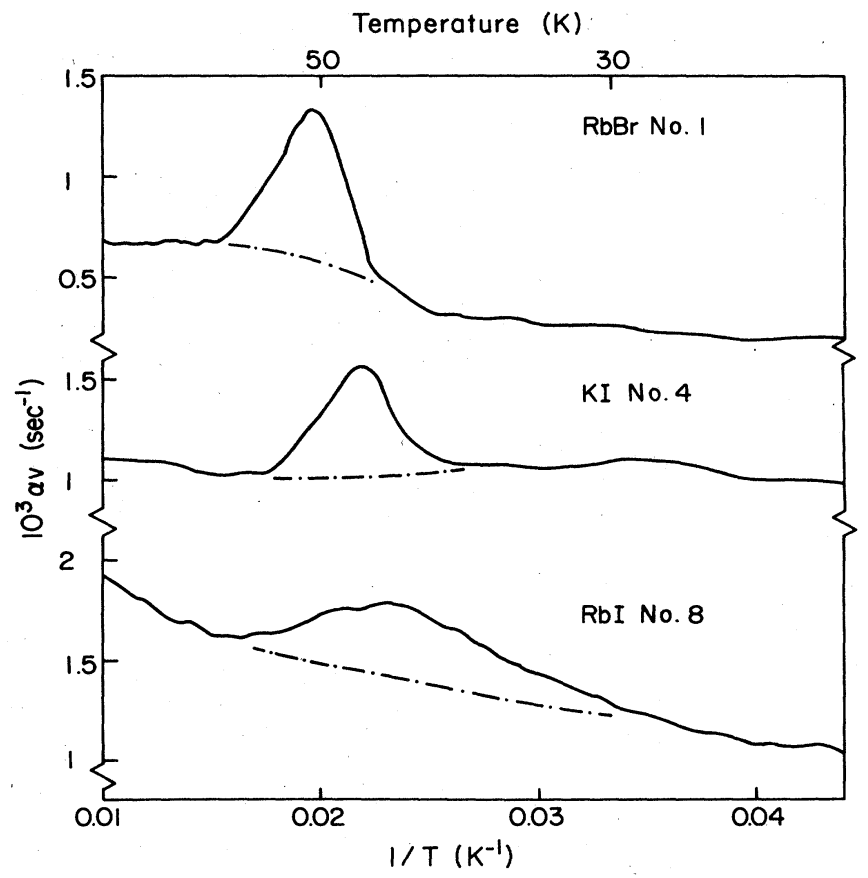

FIG. 3. Ultrasonic absorption peak near $50 \mathrm{~K}$ for three alkali halide crystals doped with $\mathrm{KReO}_{4}$. The numbers identify the samples in Table I. process between two wells with asymmetry energy $\Delta$, the relaxation time should vary with temperature as ${ }^{6}$

$$
\tau_{p}=\tau_{o p} \tanh \left(\frac{\Delta}{2 k T}\right)
$$

The two parameters $\left(\Delta=6.25 \times 10^{-2} \mathrm{meV}\right.$ and $\tau_{o p}=10^{-6}$ sec) which fit the initial increase with decreasing temperature shown in Fig. 4 cannot account for the saturation ob-

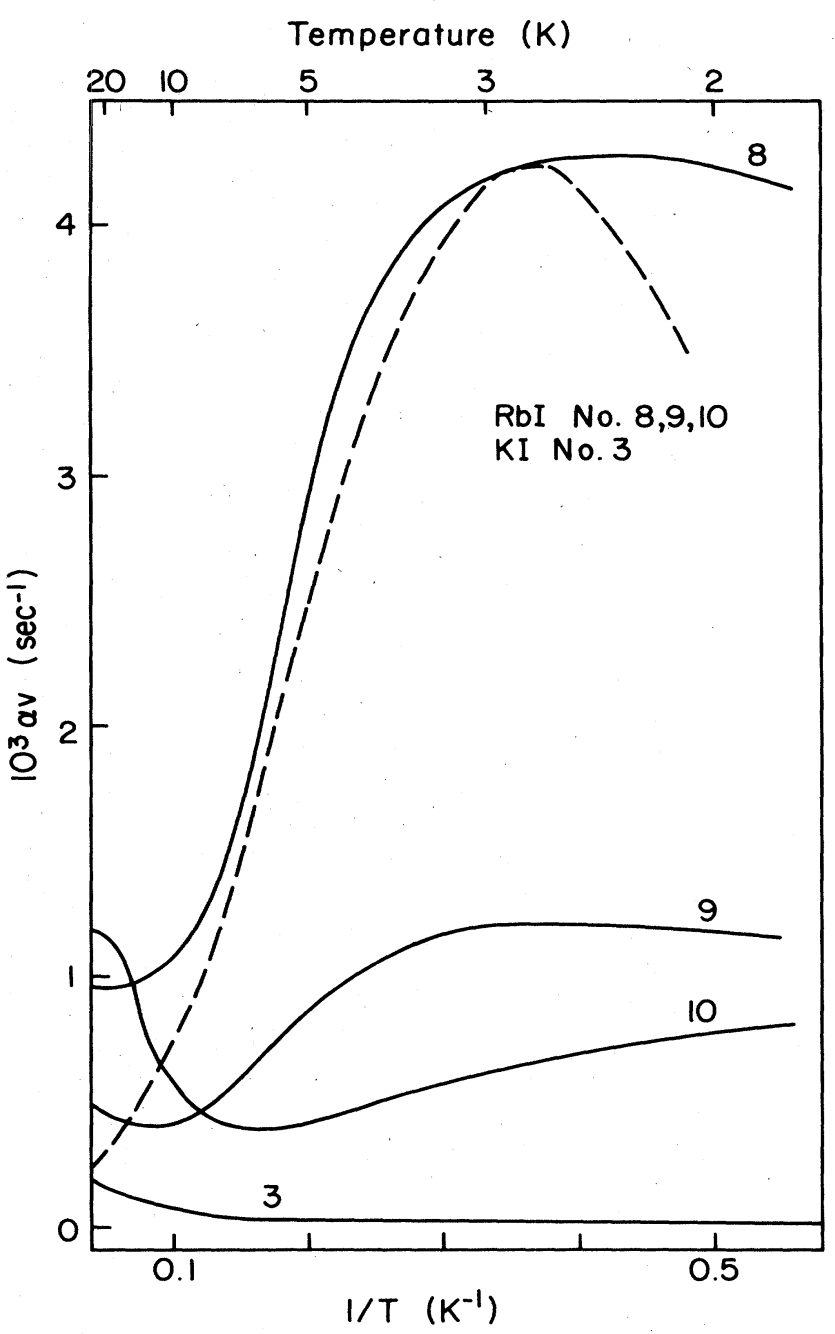

FIG. 4. Low-temperature absorption in $\mathrm{RbI}: \mathrm{KReO}_{4}$. The dashed line is calculated assuming an activation process for this degree of freedom. The numbers identify the samples in Table I. 
served at the lowest temperatures. Although this nearlytemperature-independent absorption can be fitted by introducing more parameters into the relaxation model (e.g., a distribution of energy values in the activation process) no additional insights are uncovered.

We now compare the ultrasonic results with the persistent ir hole burning data. ${ }^{1}$ The lifetimes for persistent spectral holes and antiholes in the $\nu_{3}$ mode of $\mathrm{ReO}_{4}{ }^{-}$in these alkali halides depend strongly on temperature. Earlier measurements have shown that when a sample containing a longlived feature is cycled to roughly $6 \mathrm{~K}$ and back down to 1.4 $\mathrm{K}$, roughly $30 \%$ of the feature anneals away. ${ }^{1}$ Although accurate temperature-jump experiments have not yet been possible, it appears that the hole lifetime is much greater than $1 \mathrm{~min}$ at $-6 \mathrm{~K}$ and much less than 1 min at $10 \mathrm{~K}$. Clearly both the activation and phonon-assisted tunneling times obtained from the low-temperature data in Fig. 4 are too short to account for any persistent effects; however, the high-temperature activation relaxation time does give reasonable values. Inserting the parameters $E$ and $\tau_{0 a}$ from
Table II into Eq. (3), one obtains that spectral features should erase within several seconds at a temperature $T_{\text {er }} \sim 12 \mathrm{~K}$ from RbI while for KI, a temperature $T_{e r} \sim 17 \mathrm{~K}$ is obtained. These estimates are in qualitative agreement with the experimental results ${ }^{1}$ of about $10 \mathrm{~K}$.

A prediction from our ultrasonic measurements, which would conclusively link the optical memory properties to the observed activation process, is that the spectral features should be more persistent for KI than for RbI. An important future study would be the thorough investigation of the lifetime of persistent ir spectral features for $\mathrm{ReO}_{4}^{-}$as a function of temperature for different host lattices.

This work has been supported by U.S. Army Research Office Grants No. DAAG-29-83-K-0044 and No. DAAG-2984-K-0034 and National Science Foundation Grant No. DMR-79-28008 through the Cornell Materials Science Center. One of us (H.L.) acknowledges the receipt of financial support from the Deutsche Forschungsgemeinschaft.
"Present address: Institut für Angewandte Physik, Universität, 8400 Regensburg, West Germany.

1W. E. Moerner, A. R. Chraplyvy, A. J. Sievers, and R. H. Silsbee, Phys. Rev. B 28, 7244 (1983).

${ }^{2}$ T. R. Gosnell, A. J. Sievers, and R. H. Silsbee, Phys. Rev. Lett. 52, 303 (1984).

${ }^{3}$ H. Lengfellner, T. R. Gosnell, R. W. Tkach, and A. J. Sievers,
Appl. Phys. Lett. 43, 437 (1983)

${ }^{4}$ See, for example, R. Truell, C. Elbaum, and B. B. Chick, Ultrasonic Methods in Solid State Physics (Academic, New York, 1969).

${ }^{5}$ A. S. Nowick and W. R. Heller, Adv. Phys. 14, 101 (1965).

${ }^{6}$ J. Jäckle, L. Pinché, W. Arnold, and S. Hunklinger, J. Non-Cryst. Solids 20, 365 (1976). 